Check for updates

1 Paul Watkiss Associates Limited, Oxford, UK

2 Center for Health and the Global Environment (CHanGE), University of Washington, USA

Cite this as: BMJ 2022;376:0313 http://dx.doi.org/10.1136/bmj.0313

Published: 04 February 2022

\section{A lack of climate finance is harming population health}

\author{
Paul Watkiss and Kristie Ebi argue that new approaches are needed to scale up financing for health \\ adaptation to climate change.
}

Paul Watkiss, ${ }^{1}$ Kristie L Ebi ${ }^{2}$

Recent climate change already has negative effects on human health and projected climate change is expected to contribute to substantial increases in morbidity and mortality associated with a range of health outcomes. ${ }^{1}$ The two main policy responses are mitigation to reduce global emissions of greenhouse gases to decrease the magnitude of health risks later this century, and adaptation to prepare for and minimize the negative health impacts of climate change.

National planning on climate change is advancing. As part of the United Nations Framework Convention on Climate Change process, countries are assessing climate related risks and needs, and are developing mitigation and adaptation plans, including for health. ${ }^{2}$ The Paris Agreement requires countries to submit Nationally Determined Contributions (NDCs) every five years. These set out priorities and commitments to reduce national emissions and adapt to the impacts of climate change. Health is reported as a priority in over $50 \%$ of NDCs that feature adaptation. ${ }^{3}$ As part of the World Health Organisation 2021 Health and Climate Change Global Survey, ${ }^{2}$ approximately two thirds of the 95 respondent countries reported undertaking a climate change and health vulnerability and adaptation assessment.

Global finance flows for mitigation and adaptation plans has also been increasing. ${ }^{4}$ Climate finance for mitigation is substantial, with an annual average of USD 571 billion in 2019/2020. This includes public and private finance. Global finance for adaptation across all sectors is only a fraction of this, at USD 46 billion in the same period and it is almost all public. ${ }^{4}$ In 2017/2018 this adaptation finance was mainly prioritised to three sectors: water management (32\%), agriculture and land use (24\%), and disaster risk management (22\%). 5 Finance flows to health sector activities are extremely low, receiving less than one percent of climate adaptation finance, ${ }^{3}$ a tiny fraction of the estimated level of funding required to minimise negative health outcomes.

There are several reasons why a disconnect exists, i.e., why health is identified as an important climate risk in national plans but is not being prioritized for adaptation finance. At the national level, prioritisation of adaptation financing tends to be targeted towards climate or environment ministries, or sectors perceived to be at high risk, such as agriculture. Health may be seen as a lower priority because it does not lead to immediate economic benefits or growth. In addition, health ministries face several barriers to accessing climate finance, including a lack of information on climate financing opportunities, a disconnect of health actors to climate change processes, and limited capacity to prepare country proposals for health and climate change activities. ${ }^{2}$ In low and middle income countries, insufficient domestic budgets in the health sector compound these issues.

These challenges are not insurmountable. International multilateral and bilateral donors can play an important role in supporting the development of adaptation measures in countries through financing, capacity building, and technical assistance. This includes increased funding for analysing the climate risks to health and identifying adaptation responses as part of developing national adaptation plans (NAPs). Health NAPs are also needed, which include costed health interventions. Learning from best practice and country experiences shows that such plans can prioritize action to address the health impacts of climate change at all levels of planning and promote the co-benefits of mitigation and adaptation in other sectors. ${ }^{6}$

There is also a need to mainstream climate adaptation into national and sector development plans, integrating it into medium term plans and annual budgets for health. Certain factors are associated with successful integration, including a high level champion, buy in of the relevant planning or finance ministries, and technical assistance and capacity building within health ministries, as well as finance. ${ }^{7}$ Mainstreaming climate change adaptation in health plans can leverage development budgets and nudge sector development along climate smart pathways. It can also help health ministries to access bilateral funding (e.g. sector budget support, health programming) and global finance, such as development loans for hospital infrastructure investment.

New financial models and approaches could also be explored to increase financing for health adaptation. For example, there are opportunities to encourage or co-finance with investors looking to create positive social and environmental impacts alongside financial returns, including philanthropic funders, and to raise finance from private investors and financial markets. There is also potential to attract private investment with blended finance e.g., using public funds to de-risk. Finally, there may be opportunities to integrate climate adaptation into existing public private partnerships within the health sector. ${ }^{8}$

Health is a key priority for climate change adaptation but has not received the attention or funding it requires. Addressing this gap requires a greater focus on adaptation from development partners, as well as a combination of capacity support, sector 


\section{OPINION}

\section{mainstreaming, and innovative approaches to finance.}

Competing interests: none declared

Acknowledgment: This paper is part of the BMJ collection launched at the Prince Mahidol Award Conference 2022 "The World We Want: Actions Towards a Sustainable, Fairer and. Healthier Society"

Provenance and peer review: commissioned, not peer reviewed

1 Haines A, Ebi K. The imperative for climate action to protect health. N Engl J Med 2019;380:263-73doi: 10.1056/NEJMra1807873.

2 World Health Organization. (2021). 2021 WHO health and climate change global survey report. Geneva: World Health Organization; 2021. Licence: CC BY-NC-SA 3.0 IGO.

https://www.who.int/publications/i/item/9789240038509

3 UNEP. Adaptation Gap Report 2018. United Nations Environment Programme (UNEP), 2018, Nairobi, Kenya.

4 CPI. Global Landscape of Climate Finance 2020. Climate Policy Initiative. 2020. https://www.climatepolicyinitiative.org/wp-content/uploads/2021/10/Global-Landscape-of-ClimateFinance-2021.pdf

5 CPI. Global Landscape of Climate Finance 2019. Climate Policy Initiative. 2019. November, 2019 https://www.climatepolicyinitiative.org/wp-content/uploads/2019/11/2019-Global-Landscape-ofClimate-Finance.pdf

6 Quality criteria for health national adaptation plans. Geneva: World Health Organization; 2021. https://www4.unfccc.int/sites/NAPC/Documents/Supplements/WHO\%20Criteria\%20for\%20quality\%20NAPs.pdf

$7 \quad$ WRI From Planning to Action: Mainstreaming Climate Change Adaptation into Development. World Resources Institute, 2018. Working Paper https://www.wri.org/research/planning-actionmainstreaming-climate-change-adaptation-development

8 Frisari GL, Mills A, Zuniga S, et al. Pohl let al. Climate Resilient Public Private Partnerships: A Toolkit for Decision Makers. Washington DC. Inter-American Development Bank. https://publications.iadb.org/en/climate-resilient-public-private-partnerships-a-toolkit-for-decision-makers 